\title{
Effects of hyperbaric oxygen on vascular endothelial function in patients with slow coronary flow
}

\author{
Yuan $\mathrm{Li}^{1}$, Huiping Zhang ${ }^{2}$, Yi Liang ${ }^{3}$, Wei Wang ${ }^{3}$, Tongshun $\mathrm{Xu}^{1}$, \\ Jifang Zhang ${ }^{1}$, Wenliang Xiao ${ }^{4}$, Tao Wang ${ }^{4}$ \\ ${ }^{1}$ Department of Cardiology, The Fourth Hospital of Shijiazhuang, China \\ ${ }^{2}$ Department of Otolaryngology, Heibei People's Hospital, China \\ ${ }^{3}$ Department of Hyperbaric Oxygen Therapy, the Third Hospital of Hebei Medical University, China \\ ${ }^{4}$ Department of Cardiology, the Third Hospital of Hebei Medical University, China
}

\begin{abstract}
Background: To improve therapy for slow coronary flow (SCF), the effects of hyperbaric oxygen (HBO) therapy on vascular endothelial function in SCF patients is the focus of this investigation.

Methods: Ninety-eight patients who exhibited chest discomfort were retrospectively analyzed, and diagnosed with SCF by coronary artery angiography at the Third Hospital of Hebei Medical University, Shijiazhuang, China from 2014 to 2016. The patients were divided into two groups according to the following treatment: $H B O$ group $(n=48)$ and the control group $(n=50)$. Patients in the control group were administrated with conventional treatment, while those in the HBO group were administrated $H B O$ therapy for 4 weeks in addition to conventional treatment. To evaluate the effects of $H B O$ on vascular endothelial functions, plasma levels of nitric oxide (NO), calcitonin gene-related peptide (CGRP), endothelin-1 (ET-1), high sensitivity C-reactive protein (hsCRP) as well as endothelial-dependent flowmediated vasodilation (FMD) of the brachial artery were measured in both groups before and after their respective treatments.

Results: There were no significant differences in plasma levels of NO, ET-1, CGRP, hsCRP nor in $F M D$ measurements between the two groups before treatment $(p>0.05)$. Moreover, the levels of all the parameters measured showed no significant changes before and after treatment in the control group. However, when comparing the control group, FMD and plasma NO and CGRP levels were significantly increased in the HBO group after treatment $(p<0.01)$, whereas hsCRP and ET-1 levels decreased dramatically $(p<0.001)$.

Conclusions: The HBO treatment in addition to conventional therapy may significantly improve the vascular endothelial function in SCF patients. (Cardiol J 2018; 25, 1: 106-112)
\end{abstract}

Key words: slow coronary flow, hyperbaric oxygen, vascular endothelial function

\section{Introduction}

Slow coronary flow (SCF) is the phenomenon characterized by the slow passage of contrast in the absence of obstructive coronary artery diseases, including coronary artery coronary spasm, thrombosis, coronary artery dilatation, heart valve disease and connective tissue disease [1]. Patients with SCF often exhibit angina pectoris or acute coronary syndrome, have high rates of re-hospitalization and are more likely to be diagnosed with other cardiovascular-related diseases [2,3].

Hyperbaric oxygen (HBO) therapy is defined as subjecting patients to oxygen breathing at ambient temperatures which are greater than normal atmospheric pressure. It has been demonstrated that increasing oxygen partial pressure and blood oxygen levels, enhances tissue oxygen dispersion,

Address for correspondence: Dr. Tao Wang, Department of Cardiology, the Third Hospital of Hebei Medical University, China, tel: +86-13582102651, e-mail: docwt781130@163.com

Received: 30.06.2017 Accepted: 22.10.2017 
and increases blood flow in the ischemic area as well as improves myocardial aerobic metabolism and energy metabolism [4]. It can also decrease the heart rate, and reduce myocardial contractility so that myocardial oxygen consumption will be decreased and relieve the symptoms of hypoxia myocardium [4].

In this study, HBO therapy was applied on SCF patients in addition to the conventional treatments. Before and after HBO treatment, under investigation were the effects of HBO on SCF patients by examining endothelial-dependent flow-mediated vasodilation (FMD) of the brachial artery, and the plasma levels of nitric oxide (NO), endothelin-1 (ET-1), calcitonin gene-related peptide (CGRP), and high sensitivity C-reactive protein (hsCRP). These results demonstrated that HBO treatment can significantly improve the vascular endothelial functions, which might explain how HBO therapy improves the symptoms of cardiovascular diseases.

\section{Methods}

\section{Objects}

Ninety-eight patients who exhibited chest pain or chest discomfort were retrospectively analyzed, and diagnosed with SCF by coronary artery angiography in the Third Hospital of Hebei Medical University from 2014 to 2016 . This study was approved by the ethics committee of the third hospital in Hebei Medical University. Informed consent was signed by all patients who participated in this study.

\section{Diagnosis of SCF}

Judkins technique and corrected Thrombolysis in Myocardial Infarction (TIMI) frame count (CTFC) were used for the diagnosis of SCF. All the patients were evaluated by two independent experienced physicians (deputy chief physician), and average values were taken into account. Patients with CTFC values greater than normal range $(15 \leq \mathrm{CTFC} \leq 27)$ were diagnosed with SCF [5].

\section{Treatment}

Before the surgery of coronary angiography, all patients were administered conventional medications with the same dosage for anti-platelet, anticoagulation, vessel expansion, and lipid regulation according to the guide for treatment for coronary heart disease and angina (Table 1).

For the HBO group, patients who had stable hemodynamics with no arrhythmia nor angina pectoris, were administrated HBO therapy with an initial pressure of $0.2 \mathrm{MPa}$ (2ATA) for $20 \mathrm{~min}$ in a cabin. Next, patients were given pure oxygen $2 \times 40 \mathrm{~min}$, with a $5-10 \mathrm{~min}$ break with cabin air $(0.2 \mathrm{MPa})$ in between. After treatment, the pressure of the cabin was decompressed in $20 \mathrm{~min}$. The whole procedure lasted about $120 \mathrm{~min}$ per treatment. Patients from the HBO group were treated using this procedure once per day and 6 times per week for 4 weeks. The patients in the control group were not treated with $\mathrm{HBO}$ therapy.

During the HBO treatment, patient's heart rate, blood pressure changes, and other symptoms of discomfort were closely monitored. In addition, several concerns should be taken into consideration for $\mathrm{HBO}$ treatment: 1 ) the pressure in the treatment should not be higher than $0.2 \mathrm{MPa}$; 2 ) and the process of compression and decompression should be slow and smooth; 3 ) the pressure regulator should be stable; 4 ) before HBO treatment, patients should be administrated with vascular smooth muscle dilatation drugs, such as isosorbide mononitrate.

\section{FMD measurement}

Before measurement, patients stopped taking any drugs which might affect the measurement. Patients avoided drinking caffeine-containing beverages and food, and had fasted for more than $4 \mathrm{~h}$.

For the measurement, the brachial artery $2-15 \mathrm{~cm}$ above the elbow was used as the target artery. The distance between the brachial artery and intima of the brachial artery was measured on the longitudinal section. The mean value was calculated by 3 measurements at the same site. The base diameter of patient brachial artery under relaxed status (D1) was determined first. Then the reactive hyperemia was measured at the distal end of the target artery. The sphygmomanometer was inflated to $300 \mathrm{~mm} \mathrm{Hg}$ pressure, and decompressed to $0 \mathrm{~mm} \mathrm{Hg}$ within $4 \mathrm{~min}$. The reactive hyperemia (D2) was measured 60-90 s after decompression. The recovered brachial artery diameter (D3) was measured after a 15 min break, while the brachial artery diameter (D4) was measured $4-5 \mathrm{~min}$ after administration with $0.5 \mathrm{mg}$ of sublingual nitroglycerin. To calculate the FMD and endothelium-independent diastolic function by measuring nitrate-mediated dilatation (NMD), the following formulas were applied:

- $\mathrm{FMD}=(\mathrm{D} 2-\mathrm{D} 1) / \mathrm{D} 1 \times 100 \%$

- $\mathrm{NMD}=(\mathrm{D} 3-\mathrm{D} 4) / \mathrm{D} 4 \times 100 \%$

All operations were performed blindly by two experienced, high-grade B-ultrasonographers, and the mean values were calculated. 
Table 1. Comparison of general clinical conditions between the two groups of patients.

\begin{tabular}{|c|c|c|c|c|}
\hline Medication & Dosage & Effect & Mechanisms & Company \\
\hline Aspirin & 100 mg/day & $\begin{array}{l}\text { Inhibition of platelet } \\
\text { aggregation }\end{array}$ & $\begin{array}{l}\text { Block the formation } \\
\text { of thromboxane } A_{2} \\
\text { in platelets }\end{array}$ & $\begin{array}{c}\text { Bayer Healthcare Co. Ltd., } \\
\text { Germany }\end{array}$ \\
\hline Ticagrelor & $180 \mathrm{mg} /$ day & $\begin{array}{l}\text { Inhibition of platelet } \\
\text { aggregation }\end{array}$ & $\begin{array}{l}\text { An antagonist against } \\
\mathrm{P} 2 \mathrm{Y}_{12} \text { receptor }\end{array}$ & $\begin{array}{c}\text { AstraZeneca } \\
\text { Pharmaceutical Co. Ltd., UK }\end{array}$ \\
\hline $\begin{array}{l}\text { Metoprolol } \\
\text { tartrate }\end{array}$ & $50 \mathrm{mg} /$ day & $\begin{array}{l}\text { Reduce heart rate; } \\
\text { relieve the chest pain } \\
\text { due to poor blood flow }\end{array}$ & $\beta_{1}$ receptor blocker & $\begin{array}{c}\text { AstraZeneca } \\
\text { Pharmaceutical Co. Ltd., UK }\end{array}$ \\
\hline $\begin{array}{l}\text { Isosorbide } \\
\text { mononitrate }\end{array}$ & 40 mg/day & $\begin{array}{l}\text { Dilate the blood } \\
\text { vessels to reduce } \\
\text { the blood pressure; } \\
\text { reduce the occurrence } \\
\text { of angina }\end{array}$ & $\begin{array}{l}\text { Nitrate-class drug to } \\
\text { release nitric oxide, } \\
\text { which increases } \\
\text { cGMP level }\end{array}$ & $\begin{array}{l}\text { Lunan Beite } \\
\text { Pharmaceutical Co. Ltd., } \\
\text { China }\end{array}$ \\
\hline Atorvastatin & $20 \mathrm{mg} /$ day & $\begin{array}{l}\text { Hypolipidemic; } \\
\text { improve the vascular } \\
\text { endothelial function }\end{array}$ & $\begin{array}{l}\text { Inhibit HMG-CoA } \\
\text { reductase, which plays } \\
\text { essential roles in } \\
\text { production of cholesterol }\end{array}$ & $\begin{array}{l}\text { Pfizer Pharmaceuticals Ltd., } \\
\text { USA }\end{array}$ \\
\hline
\end{tabular}

\section{Measurement of NO, ET-1, CGRP and hsCRP}

The plasma levels of ET- 1 and hsCRP were measured by enzyme-linked immunosorbent assay (ELISA; ET-1 kit: Shanghai Yuanye Biological Technology Co., Ltd; HsCRP assay: Shanghai Hengyuan Biological Technology Co., Ltd). NO levels were measured using a nitric acid reductase assay (Nanjing completed the Institute of Bioengineering). The expression of CGRP was measured by radioimmunoassay (Institute of East Asian Immunology, PLA General Hospital). All experiments were performed according to manufacturer's instructions.

\section{Statistical analysis}

To perform statistical analysis, SPSS16.0 was applied. The data is presented as mean \pm standard deviation $(\bar{x} \pm S)$. For comparison of the same group, student paired t-test was used, while for comparisons between the two groups the grouped t-test was applied. $\chi^{2}$ analysis was applied and statistical significance was set at $\mathrm{p}$ value $<0.05$.

\section{Results}

Comparison of general clinical conditions between the two groups of patients

Ninety-eight SCF patients were divided into two groups: the HBO group (22 females and 26 males, age: $42-69 ; 57.96 \pm 10.26)$, and the control group (23 females and 27 males, age: $42-71$; $56.26 \pm 9.52)$. No significant difference was found in sex, age, body mass index, family history of coronary heart disease, fasting blood glucose levels, blood pressure and heart rate between the two groups (Table 2). No significant difference was found in CTFC between the HBO group $(39.5 \pm 8.2 ; \mathrm{n}=48)$ and control group (37.3 $\pm 9.6 ; \mathrm{n}=50)$ (Table 2$)$. None of the patients were diagnosed with coronary artery dissection, coronary aneurysm, hypertension 2 and above, myocardial infarction, coronary thrombosis, cardiac insufficiency, liver and kidney dysfunction nor had they had any signs of infection. Furthermore, none of the patients were diagnosed with pneumothorax, severe emphysema, open empty tuberculosis with hemoptysis history, retinal detachment, uncontrolled visceral bleeding, Eustachian tube obstruction or any other hyperbaric oxygen contraindications.

\section{FMD and NMD measurements}

Since SCF patients have been reported to suffer from endothelial dysfunction [6, 7], to evaluate the effects of $\mathrm{HBO}$ on SCF patients are endothelium-dependent or independent, FMD and NMD levels of patients were measured before and after HBO treatment. No remarkable difference in FMD was observed between HBO and control groups before treatment (Fig. 1A). Moreover, no significant difference was found in FMD before and after treatment in the control group (Fig. 1A). However, interestingly, after HBO therapy, patients from the HBO group showed significantly higher level of FMD compared to patients from the control group after treatment 
Table 2. Comparison of baseline clinical conditions between two groups of patients.

\begin{tabular}{lccc}
\hline Patients & HBO & Control & P \\
\hline Sex (male/female) & $26 / 22$ & $27 / 23$ & 0.987 \\
Ages & $57.96 \pm 10.26$ & $56.26 \pm 9.52$ & 0.4978 \\
Body mass index $\left[\mathrm{kg} / \mathrm{m}^{2}\right]$ & $27.80 \pm 2.99$ & $28.39 \pm 3.78$ & 0.3948 \\
Family history of CAD (yes/no) & $16 / 32$ & $19 / 31$ & 0.630 \\
Fasting blood glucose [mmol/L] & $5.67 \pm 0.73$ & $5.49 \pm 0.69$ & 0.2126 \\
Blood level of TC [mmol/L] & $4.59 \pm 0.77$ & $4.80 \pm 0.61$ & 0.1370 \\
Blood level of LDL-C [mmol/L] & $2.91 \pm 0.68$ & $3.02 \pm 0.65$ & 0.4150 \\
Blood level of HDL-C [mmol/L] & $1.88 \pm 0.46$ & $1.91 \pm 0.35$ & 0.7165 \\
Heart rate [bpm] & $67 \pm 10$ & $71 \pm 11$ & 0.0630 \\
Hypertension & $17(35.42 \%)$ & $19(38.00 \%)$ & 0.791 \\
History of smoking & $15(31.25 \%)$ & $17(34.00 \%)$ & 0.772 \\
Corrected TIMI frame count & $39.5 \pm 8.2$ & $37.3 \pm 9.6$ & 0.2219 \\
\hline
\end{tabular}

HBO - hyperbaric oxygen; CAD - coronary heart disease; TC - total cholesterol; LDL-C — low-density lipoprotein cholesterol; HDL-C — high-density lipoprotein cholesterol; TIMI — Thrombolysis in Myocardial Infarction

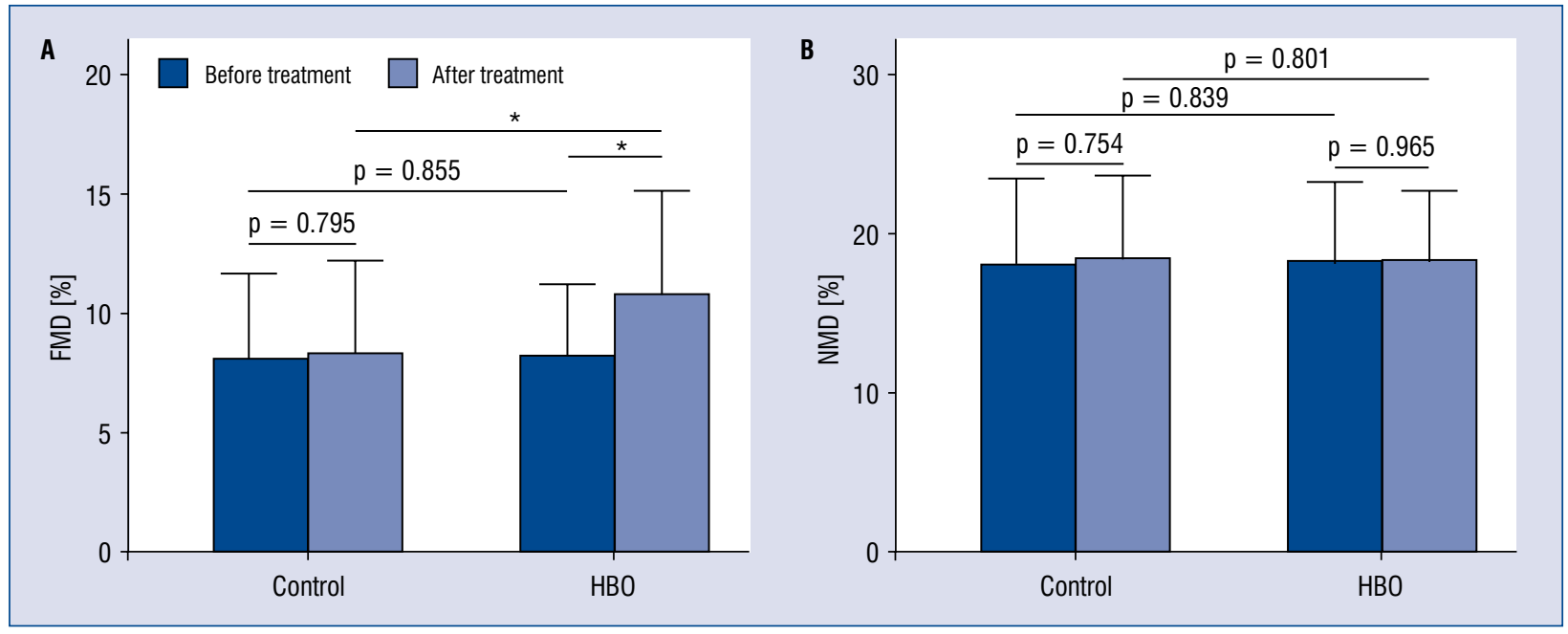

Figure 1. Flow-mediated vasodilation $(\mathbf{A})$ and nitrate-mediated dilatation (B) measurements between control and hyperbaric oxygen (HBO) groups; ${ }^{*} p<0.01$.

$(10.86 \pm 4.26$ vs. $8.39 \pm 3.81 ; \mathrm{p}<0.01$; Fig. $1 \mathrm{~A})$. To specify the effects of HBO treatment on endothelium-dependent function, NMD was also measured, which increases the coronary flow in an endothelium-independent manner. No significant difference in NMD was found before and after treatment between groups, indicating that HBO treatment improves vascular endothelial functions (Fig. 1B).

\section{Plasma NO, ET-1, CGRP and hsCRP measurements}

To further investigate the potential mechanisms of HBO treatment on vascular endothe- lial functions, plasma levels of NO [8], ET-1 [8], CGRP [9] were analyzed, these were considered as biomarkers for vascular endothelial functions, and hsCRP, a maker of the inflammatory response $[10,11]$. There were no significant differences in plasma levels of NO, ET-1, CGRP and hsCRP between the groups before treatment. After treatment, compared to control patients, the patients who undertook HBO therapy exhibited significantly higher plasma levels of $\mathrm{NO}(32.28 \pm 9.24 \mathrm{vs}$. $26.50 \pm 8.95 \mu \mathrm{M} ; \mathrm{p}<0.01$; Fig. 2A) and CGRP $(30.78 \pm 8.23$ vs. $25.96 \pm 6.28 \mathrm{pg} / \mathrm{mL} ; \mathrm{p}<0.01$; Fig. 2B), and significant reductions of ET-1 (14.46 \pm \pm 6.04 vs. $19.57 \pm 6.51 \mathrm{pg} / \mathrm{mL} ; \mathrm{p}<0.001$; Fig. $2 \mathrm{C}$ ) 

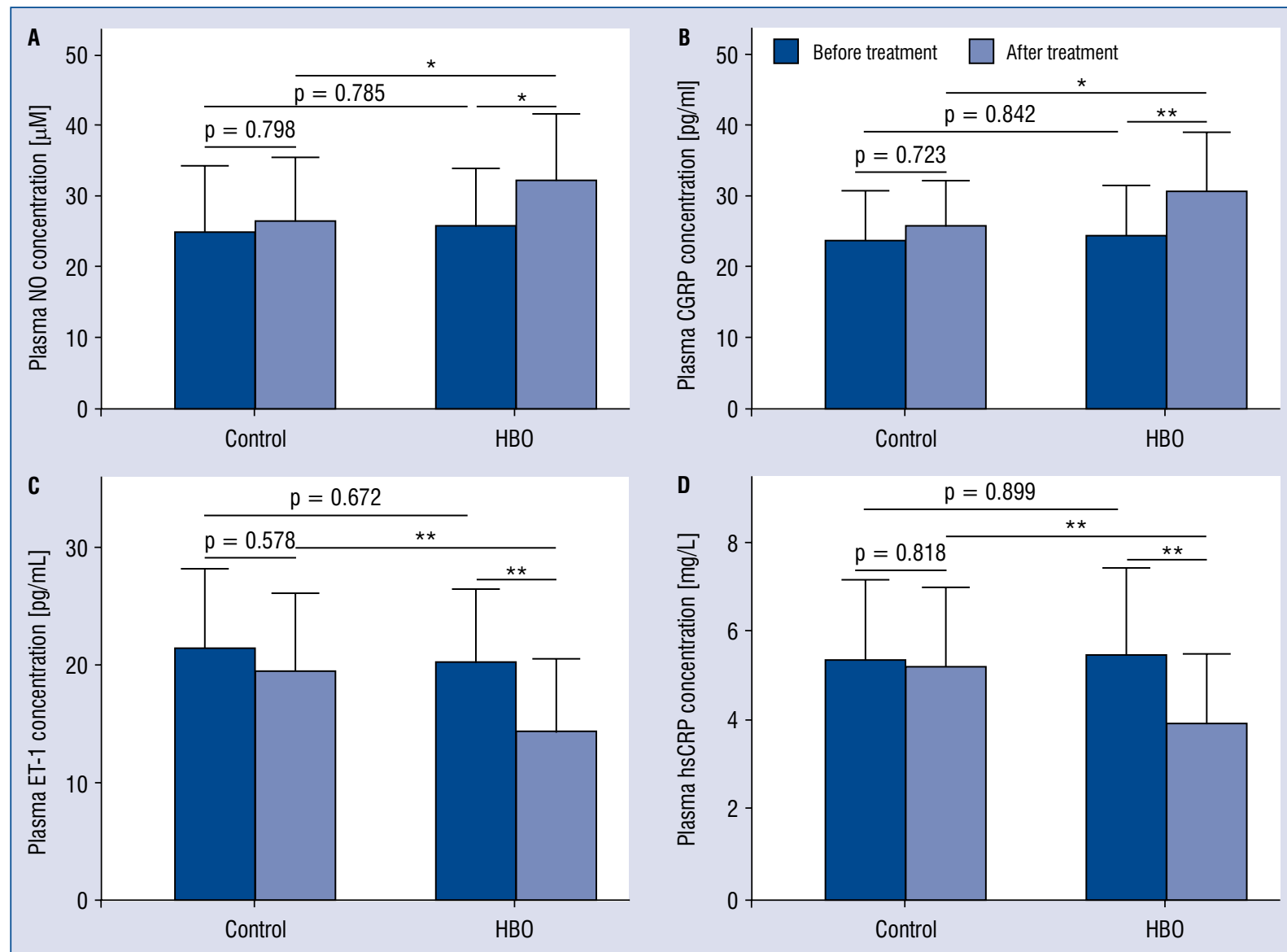

Figure 2. Plasma nitric oxide (NO) (A), calcitonin gene-related peptide (CGRP) (B), endothelin-1 (ET-1) (C), and high sensitivity $\mathrm{C}$-reactive protein ( $\mathrm{hsCRP}$ ) (D) were measured in control and hyperbaric oxygen groups; ${ }^{*} p<0.01 ;{ }^{* *} p<0.001$.

and hsCRP $(3.93 \pm 1.56$ vs. $5.21 \pm 1.79 \mathrm{mg} / \mathrm{L}$; p $<0.001$; Fig. 2D) levels. No significant differences in plasma levels of NO, ET-1, CGRP and hsCRP were observed before and after treatment in the control group (Fig. 2A-D). These results further confirmed the effects of $\mathrm{HBO}$ treatment on vascular endothelial functions.

\section{Discussion}

Slow coronary flow affects $1-7 \%$ of patients who are tested by angiography due to clinical suspicion of cardiovascular diseases [12]. The pathological mechanisms underlying SCF are still unclear. Accumulating evidence suggests that microvascular lesions or functional abnormities are associated with the onset of SCF [13]. Pekdemir et al. [8] reported an imbalanced released of ET-1 and NO in SCF patients. In particular, ET-1 levels were exceptionally high in SCF patients. These results imply that SCF patients may suffer from impair- ment of endothelial function. Indeed, in another independent study, Signori et al. [6] claimed that SCF patients suffered from endothelial dysfunction in both arteries and veins, and implied that $\mathrm{SCF}$ could be a global vascular phenomenon. Furthermore, they found that the level of $\mathrm{C}$-reactive protein, an independent risk factor for SCF, is significantly higher in SCF patients. In addition, Yoon et al. [7] observed lower FMD values, which is also considered as an independent risk factor for SCF in patients. It decreased even before the thickening of the carotid intima. Taken together, vascular endothelial dysfunction is thought to be closely associated with the pathogenesis of SCF.

Flow-mediated vasodilation is widely accepted as a non-invasive method for vascular endothelial function evaluation and an indicator for early coronary lesions [14]. NO and ET-1 are also considered biomarkers for vascular endothelial function. As the main diastolic vascular factor, CGRP interacts with its receptors on vascular endothelial cells, which 
in turn enhances intracellular calcium levels via the cyclic adenosine monophosphate pathway [9]. This leads to enhanced activity of NO synthase, which further increases the vasodilatory effects of NO. Furthermore, hs-CRP, a maker of the inflammatory response involved in many types of acute and chronic physiological inflammation, is thought to be closely related to SCF onset $[10,11]$.

According to Bennett's report [15], HBO treatment can reduce myocardial ischemia and mortality rate of patients suffering from acute coronary syndrome. Another study from Khan et al. [16] showed that HBO treatment can increase myocardial oxygen supply, and enhance the expression of vascular endothelial growth factors and the recovery of cardiomyocytes, and can also significantly improve the efficacy of stem-cell transplantationtreated myocardial infarct rats. Previous study also revealed that HBO treatment improved the symptoms of diabetic patients might be due to the effects of HBO on vascular functions. However, the potential mechanism is still unclear [17]. In addition, there are few reports on the effects of $\mathrm{HBO}$ treatment in improving vascular endothelial function of SCF patients.

In this study, in addition to regular, conventional treatments, SCF patients were administrated with HBO therapy. As a result, patients who received HBO treatment showed remarkably better FMD values and have significantly higher $\mathrm{NO}$ and CGRP levels, while lower ET-1 and hs-CRP levels compared to control SCF patients who received only conventional therapy. These data imply that HBO treatment could reduce patient vascular inflammatory response and, at the same time, reduce the expression of vasoconstrictor factors, such as ET-1, and also increase the expression of vasodilators, including NO and CGRP, in reducing SCF symptoms. However, no significant change was observed in NMD measurement, which indicated the endothelium-independent diastolic function, further indicating that HBO therapy improves the symptom of SCF patients by enhancing the vascular endothelial functions.

In addition to its oxygen supply mechanism, HBO treatment can also exert influence in many other aspects: 1) Anti-oxidizing effects. Li et al. [18] found that HBO can improve the activity of catalase (CAT) and superoxide dismutase (SOD), while reducing the production of malondialdehyde (MDA) and large amounts of oxygen free radicals produced during ischemia-reperfusion; 2) Inhibit inflammation. According to Cheng et al. [19], HBO therapy can inhibit cyclooxygenase-2 (COX-2) and reduce the synthesis of prostaglandin (PG) and thromboxane A2 (TXA2); 3) HBO has been demonstrated to increase the expression of various fibrinolytic factors in vascular endothelial cells, and reduce blood viscosity thus altering blood rheology [20]; 4) Promote the formation of collateral circulation [21]; 5) Inhibit the development of atherosclerosis [22].

In a previous study, simvastatin was administered for 6 months to treat SCF patients [23]. Analysis using single photon emission computed tomography revealed a significant improvement in SCF, implying that simvastatin may exhibit statinslike multi-efficacy benefits in blood vessels and cardiomyocytes in addition to its hypolipidemic effects. Caliskan et al. [24] also reported that atorvastatin improves the microvascular function of the coronary artery and increase coronary blood flow in SCF patients. However, in this study, SCF patients from the control group were administrated with statins, but no improvement was observed, this may be due to the relatively short-term administration of the drugs. Further investigation is required to evaluate the efficacy of stains in changing endothelial function of SCF patients.

\section{Limitation of the study}

Noteworthy, HBO therapy still has many disadvantages which require further study. First, $\mathrm{HBO}$ treatment requires special equipment, which limits its daily practice for SCF patients. Second, long-term effects of HBO treatment, for example harmful oxidative stress on vascular function are still unclear and needs further study. It would be valuable to examine different conditions of $\mathrm{HBO}$ treatment and combination treatment to demonstrate the most effective, while also minimizing harmful conditions for HBO therapy of SCF patients.

\section{Conclusions}

Taken together, HBO treatment can significantly improve endothelial function of SCF patients, and may be therapeutically beneficial to SCF prognosis. In this study, a limited sample set was investigated and measurements were recorded over a relatively short-term period. Further investigation should be carried out with long-term prognostic follow-up of the patients.

\section{Acknowledgements}

This work is supported by the Key Science and Technology Planning Project of Health Bureau of 
Hebei Province, China (20120173). Contribution of the members participating in this study are appreciated, as well as the psychiatrists who helped with diagnoses.

\section{Conflict of interest: None declared}

\section{References}

1. Tambe AA, Demany MA, Zimmerman HA, et al. Angina pectoris and slow flow velocity of dye in coronary arteries: a new angiographic finding. Am Heart J. 1972; 84(1): 66-71, indexed in Pubmed: 5080284.

2. Singh S, Kothari SS, Bahl VK. Coronary slow flow phenomenon: an angiographic curiosity. Indian Heart J. 2004; 56(6): 613-617, indexed in Pubmed: 15751515.

3. Fragasso G, Chierchia SL, Arioli F, et al. Coronary slow-flow causing transient myocardial hypoperfusion in patients with cardiac syndrome X: long-term clinical and functional prognosis. Int J Cardiol. 2009; 137(2): 137-144, doi: 10.1016/j.ijcard.2008.06.070, indexed in Pubmed: 18762343.

4. Gesell, Laurie B. Hyperbaric Oxygen Therapy Indications. The Hyperbaric Oxygen Therapy Committee Report (12th ed.) 2008. Durham, NC: Undersea and Hyperbaric Medical Society.

5. Gibson CM, Cannon CP, Daley WL, et al. TIMI frame count: a quantitative method of assessing coronary artery flow. Circulation. 1996; 93(5): 879-888, indexed in Pubmed: 8598078.

6. Signori LU, Quadros AS, Sbruzzi G, et al. Endothelial function in patients with slow coronary flow and normal coronary angiography. Clinics (Sao Paulo). 2012; 67(6): 677-680, indexed in Pubmed: 22760911.

7. Yoon HJu, Jeong MHo, Cho SH, et al. Endothelial dysfunction and increased carotid intima-media thickness in the patients with slow coronary flow. J Korean Med Sci. 2012; 27(6): 614-618, doi: 10.3346/jkms.2012.27.6.614, indexed in Pubmed: 22690091.

8. Pekdemir H, Polat G, Cin VG, et al. Elevated plasma endothelin-1 levels in coronary sinus during rapid right atrial pacing in patients with slow coronary flow. Int J Cardiol. 2004; 97(1): 35-41, doi: 10.1016/j.ijcard.2003.06.025, indexed in Pubmed: 15336804 .

9. Nikitenko LL, Blucher N, Fox SB, et al. Adrenomedullin and CGRP interact with endogenous calcitonin-receptor-like receptor in endothelial cells and induce its desensitisation by different mechanisms. J Cell Sci. 2006; 119(Pt 5): 910-922, doi: 10.1242/ jcs.02783, indexed in Pubmed: 16495482.

10. Yurtdaş M, Yaylali YT, Kaya Y, et al. Increased plasma highsensitivity C-reactive protein and myeloperoxidase levels may predict ischemia during myocardial perfusion imaging in slow coronary flow. Arch Med Res. 2014; 45(1): 63-69, doi: 10.1016/j. arcmed.2013.10.019, indexed in Pubmed: 24316393.

11. Cetin M, Zencir C, Tasolar H, et al. The association of serum albumin with coronary slow flow. Wien Klin Wochenschr. 2014;
126(15-16): 468-473, doi: 10.1007/s00508-014-0559-8, indexed in Pubmed: 24981407.

12. Hawkins BM, Stavrakis S, Rousan TA, et al. Coronary slow flow--prevalence and clinical correlations. Circ J. 2012; 76(4): 936-942, indexed in Pubmed: 22293446.

13. Erdogan D, Caliskan M, Gullu $\mathrm{H}$, et al. Coronary flow reserve is impaired in patients with slow coronary flow. Atherosclerosis. 2007; 191(1): 168-174, doi: 10.1016/j.atherosclerosis.2006.03.016, indexed in Pubmed: 16620834.

14. Liu H, Wang H. Early detection system of vascular disease and its application prospect. Biomed Res Int. 2016; 2016: 1723485, doi: 10.1155/2016/1723485, indexed in Pubmed: 28042567.

15. Bennett MH, Lehm JP, Jepson N, et al. Hyperbaric oxygen therapy for acute coronary syndrome. Cochrane Database Syst Rev. 2011(8): CD004818, doi: 10.1002/14651858.CD004818.pub3, indexed in Pubmed: 21833950.

16. Khan M, Meduru S, Pandian RP, et al. Effect of oxygenation on stem-cell therapy for myocardial infarction. Adv Exp Med Biol. 2011; 701: 175-181, doi: 10.1007/978-1-4419-7756-4_24, indexed in Pubmed: 21445785.

17. Unfirer S, Kibel A, Drenjancevic-Peric I. The effect of hyperbaric oxygen therapy on blood vessel function in diabetes mellitus. Med Hypotheses. 2008; 71(5): 776-780, doi: 10.1016/j. mehy.2008.06.016, indexed in Pubmed: 18722723.

18. Li J, Liu W, Ding S, et al. Hyperbaric oxygen preconditioning induces tolerance against brain ischemia-reperfusion injury by upregulation of antioxidant enzymes in rats. Brain Res. 2008; 1210: 223-229, doi: 10.1016/j.brainres.2008.03.007, indexed in Pubmed: 18407255.

19. Cheng O, Ostrowski RP, Wu B, et al. Cyclooxygenase-2 mediates hyperbaric oxygen preconditioning in the rat model of transient global cerebral ischemia. Stroke. 2011; 42(2): 484-490, doi: 10.1161/ STROKEAHA.110.604421, indexed in Pubmed: 21164135.

20. Tjärnström J, Holmdahl L, Falk P, et al. Effects of hyperbaric oxygen on expression of fibrinolytic factors of human endothelium in a simulated ischaemia/reperfusion situation. Scand J Clin Lab Invest. 2001; 61(7): 539-545, indexed in Pubmed: 11763412.

21. Yogaratnam JZ, Laden G, Madden LA, et al. Hyperbaric oxygen: a new drug in myocardial revascularization and protection? Cardiovasc Revasc Med. 2006; 7(3): 146-154, doi: 10.1016/j. carrev.2006.04.006, indexed in Pubmed: 16945821.

22. Kudchodkar BJ, Wilson J, Lacko A, et al. Hyperbaric oxygen reduces the progression and accelerates the regression of atherosclerosis in rabbits. Arterioscler Thromb Vasc Biol. 2000; 20(6): 1637-1643, indexed in Pubmed: 10845883.

23. Cakmak M, Tanriverdi H, Cakmak N, et al. Simvastatin may improve myocardial perfusion abnormality in slow coronary flow. Cardiology. 2008; 110(1): 39-44, doi: 10.1159/000109405, indexed in Pubmed: 17934268.

24. Caliskan M, Erdogan D, Gullu H, et al. Effects of atorvastatin on coronary flow reserve in patients with slow coronary flow. Clin Cardiol. 2007; 30(9): 475-479, doi: 10.1002/clc.20140, indexed in Pubmed: 17803205. 